\title{
Hot topics in transcatheter aortic valve implantation
}

\author{
Marco Barbanti ${ }^{1}$, Corrado Tamburino ${ }^{1,2}$ \& Davide Capodanno*,1 \\ ${ }^{1}$ Division of Cardiology - Cardio-Thoracic-Vascular Department, AOU 'Policlinico-Vittorio Emanuele', University of Catania, \\ Catania, Italy \\ ${ }^{2}$ ETNA Foundation, Catania, Italy \\ * Author for correspondence: Tel.: +39 095743 6201; Fax: +39 09536 2429; dcapodanno@gmail.com
}

\begin{abstract}
"Although TAVI is now a very well standardized procedure, there remain a few areas of controversy in which operators and researches still need to reach definite conclusions."
\end{abstract}

First draft submitted: 9 June 2017; Accepted for publication: 26 September 2017; Published online: 20 October 2017

Keywords: bicuspid aortic valve • durability • minimalist approach • TAVI • valve thrombosis

For years, surgical aortic valve replacement has been heralded as the standard of care for patients with severe symptomatic aortic stenosis (AS) [1]. However, global aging has led to an increased prevalence of severe AS in elderly patients with multiple comorbidities, who are poor candidates for surgery [2]. In the last 15 years, a less-invasive, catheter-based approach for the treatment of severe AS has been developed, assessed in well-designed clinical trials and made available to the worldwide clinical community. Transcatheter aortic valve implantation (TAVI) has been demonstrated to be a viable treatment for inoperable, high-risk and intermediate-risk elderly patients with severe AS [3-5]. The treatment of younger and lower risk AS populations is already in the pipeline [6]. Although TAVI is now a very well standardized procedure, there remain a few areas of controversy in which operators and researches still need to reach definite conclusions. This article will focus on four of such controversial topics: the efficacy of TAVI in bicuspid aortic valve stenosis; the frequency and clinical impact of transcatheter heart valve (THV) clinical and subclinical thrombosis; whether a minimalist approach for TAVI, besides saving resources, can lead to similar or even improved outcomes in selected patients; THV durability.

\section{TAVI in bicuspid aortic valves}

Bicuspid aortic valves have a high prevalence in younger patients with AS, but even in the elderly (i.e., $>80$ years of age), bicuspid valves comprise approximately $20 \%$ of the surgical cases [7]. Early experience with TAVI in bicuspid aortic valves demonstrated that this anatomical entity has several features that make the outcomes of TAVI more often suboptimal and less predictable: a more oval annulus shape, unequal leaflet size, heavy and uneven calcification of the leaflets and the presence of calcified raphes [8].

From a technical standpoint, selection of an appropriately sized TAVI device in bicuspid AS is similar to tricuspid AS, since both are based on annular properties. However, cusp morphology and calcium pattern are more complex in bicuspid aortic valves, which might interfere with optimal THV deployment and/or lead to malpositioning, aortic injuries and suboptimal hemodynamics with increased paravalvular leak. Bicuspid valve anatomy may also have effects on TAVI device expansion. A report of 21 patients, some of whom had postprocedural computed tomography, reported elliptical expansion of implanted TAVI devices in a number of patients. Noncircular expansion was possibly more common with self-expanding valves, perhaps as a result of the irregular shape and forces exerted on the expanding TAVI device in a bicuspid orifice [9].

Two recent studies on early generation TAVI devices showed a higher incidence of THV malposition requiring multiple THVs implantation (7.2\%) and moderate or severe PVR that was found in 28.4 and $15.9 \%$ of patients, respectively [10,11]. In a large multicenter propensity-based analysis comparing TAVI outcomes in bicuspid aortic valves versus tricuspid aortic valves, Yoon et al. [11] showed that among the early generation THV group, TAVI in patients with bicuspid AS was complicated more frequently with aortic root injury, mainly related to the Sapien 
XT implantation, implantation of more than one THV and moderate or severe paravalvular regurgitation, mainly related to the CoreValve implantation. Among the group treated with the new generation devices, however, there were no significant differences in procedural outcomes between bicuspid and tricuspid valves. This study suggested that the advancements incorporated by the new-generation TAVI devices accomplished to partially overcome some of the limitations encountered in treating bicuspid valves [11,12].

\section{Clinical \& subclinical THV thrombosis}

Latib et al. first reported the frequency, clinical presentation and management of THV thrombosis from a multicenter registry [13]. The overall incidence of symptomatic THV thrombosis was $0.6 \%$. All THV patients in the registry $(n=26)$ were symptomatic, and elevated transaortic gradients were detected in $92.3 \%$ of them. THV thrombosis presented as thickened leaflets or thrombotic apposition of leaflets in $76.9 \%$ and thrombotic mass on the leaflets in $23.1 \%$. 23 out of 26 patients (88.4\%) were successfully treated with anticoagulation; two patients underwent a transcatheter valve-in-valve procedure and one patient underwent surgical aortic valve replacement [13]. More recently, hypoattenuated leaflet thickening (HALT) and reduced leaflet motion (RELM) of bioprosthetic aortic valves associated with normal transvalvular gradients (possibly related to subclinical leaflet thrombosis) have been reported as affecting both transcatheter and surgical aortic valves. The prevalence of this finding ranged from 4 to $20 \%$ [14-16]. An interesting finding was that HALT/RELM was actually not observed in patients on anticoagulant therapy and resolved with initiation of anticoagulation [14-16]; these clues seem to further confirm that this finding is related to THV thrombosis. On one hand, in view of the available evidence, it may be reasonable to anticoagulate patients with symptomatic THV thrombosis (HALT/RELM) for 1-3 months. On the other hand, routine anticoagulation of all TAVI patients cannot be recommended at the present time; there are no conclusive data concerning clinical outcomes, and the risk of bleeding in a predominantly elderly population with multiple comorbidities could be high [17].

\section{Minimalist approach for TAVI}

TAVI has become a highly standardized and reproducible procedure, and one of the most debated issues is now whether TAVI is ready to be simplified [18]. In the vast majority of centers, TAVI is still considered a complex procedure for many reasons: the population treated is usually elderly with several comorbidities; TAVI requires significant technical expertise and a multidisciplinary 'heart team' to optimize patient screening and case selection; the procedure is often performed under general anesthesia; postprocedural hospitalization is generally quite prolonged. However, in recent years, some groups have been working on local programs, which incorporate specific pre-, peri- and post-procedural pathways aimed at simplification of the TAVI care. These approaches have potential to optimize preprocedural screening [19], dramatically reduce the invasiveness of the procedure (local anesthesia, fully percutaneous approach, elimination of intraprocedural transesophageal guidance, reduction or elimination of balloon pre-dilation) and shorten the duration of hospitalization, thus saving resources and accelerating the functional recovery of the patient [20-22]. The reduction or elimination of balloon predilation and the reduction in postprocedural length of stay are two of the most debated and studied issues. A few studies have demonstrated the feasibility and effectiveness of TAVI without balloon predilatation [23]. Similarly, early discharge after TAVI in selected patients demonstrated to not preclude the safety of the procedure, as demonstrated in several European and US experiences [20-22].

\section{THV durability}

TAVI is now progressively being offered to younger and lower-risk patients. In this particular subset, life expectancy is expected to exceed that of initial candidates to TAVI (i.e., elderly and very elderly patients at high or prohibitive surgical risk), which raised the question of long-term prosthesis durability of crucial importance. If THV durability up to 5 years is already a quite well-established reality, with very low rates of structural valve dysfunction demonstrated in large and methodologically rigorous studies [24-26], data on clinical outcomes and THV integrity at longer term follow-up remain extremely scarce. No significant increases in mean THV gradient or cases of structural valve deterioration were reported during the 5-year follow-up of the Placement of Aortic Transcatheter Valves (PARTNER) I trial [3]. Three studies have also reported outcomes after TAVI with either the Edwards SAPIEN or CoreValve bioprostheses up to 5 years [24]: two of them [5,6] did not suggest any major concerns regarding durability of THVs, with stable transprosthetic gradients over time and a rate of structural valve dysfunction of 3.4 and $4.2 \%$, 
respectively, using different definitions. A standardized definition of valve durability has been recently proposed by a European Task Force [27].

\section{Conclusion}

Transcatheter treatment of severe AS is a relatively young technique that in the last 15 years has experienced tremendous advancements in terms of procedural refinements, device technology and clinical evidence. Originally one of the most complex procedures in the landscape of interventional therapies, TAVI has turned now into an extremely standardized and simplified procedure. However, there are many open issues still remaining and raising the interest of operators and industries, among all the treatment of bicuspid valves, the real incidence and management of subclinical and clinical thrombosis and the long-term durability of transcatheter valves. The next 10 years will be extremely exciting and will probably provide definite answers to many unsolved questions.

\section{Financial \& competing interests disclosure}

M Barbanti has received speaker's honoraria from Edwards LifeSciences. C Tamburino and D Capodanno have received speaker's and consulting honoraria from Abbott Vascular. The authors have no other relevant affiliations or financial involvement with any organization or entity with a financial interest in or financial conflict with the subject matter or materials discussed in the manuscript apart from those disclosed.

No writing assistance was utilized in the production of this manuscript.

\section{References}

1. Nishimura RA, Otto CM, Bonow RO et al. 2017 AHA/ACC focused update of the 2014 AHA/ACC guideline for the management of patients with valvular heart disease: a report of the American College of Cardiology/American Heart Association Task Force on Clinical Practice Guidelines. Circulation 135(25), e1159-e1195 (2017).

2. Iung B, Cachier A, Baron G et al. Decision-making in elderly patients with severe aortic stenosis: why are so many denied surgery? Eur. Heart J. 26(24), 2714-2720 (2005).

3. Mack MJ, Leon MB, Smith CR et al. 5-year outcomes of transcatheter aortic valve replacement or surgical aortic valve replacement for high surgical risk patients with aortic stenosis (PARTNER 1): a randomised controlled trial. Lancet 385(9986), 2477-2484 (2015).

4. Leon MB, Smith CR, Mack MJ et al. Transcatheter or surgical aortic-valve replacement in intermediate-risk patients. N. Engl. J. Med. 374(17), 1609-1620 (2016).

5. Reardon MJ, Van Mieghem NM, Popma JJ et al. Surgical or transcatheter aortic-valve replacement in intermediate-risk patients. N. Engl. J. Med. 376(14), 1321-1331 (2017).

6. Capodanno D, Leon MB. Upcoming TAVI trials: rationale, design and impact on clinical practice. EuroIntervention 12(Y), Y51-Y55 (2016).

7. Roberts WC, Janning KG, Ko JM, Filardo G, Matter GJ. Frequency of congenitally bicuspid aortic valves in patients $\geq 80$ years of age undergoing aortic valve replacement for aortic stenosis (with or without aortic regurgitation) and implications for transcatheter aortic valve implantation. Am. J. Cardiol. 109(11), 1632-1636 (2012).

8. Jilaihawi $\mathrm{H}$, Chen $\mathrm{M}$, Webb J et al. A bicuspid aortic valve imaging classification for the TAVR era. JACC Cardiovasc. Imaging 9(10), 1145-1158 (2016).

9. Hayashida K, Bouvier E, Lefèvre T et al. Transcatheter aortic valve implantation for patients with severe bicuspid aortic valve stenosis. Circ. Cardiovasc. Interv. 6(3), 284-291 (2013).

10. Mylotte D, Lefevre T, Søndergaard L et al. Transcatheter aortic valve replacement in bicuspid aortic valve disease. J. Am. Coll. Cardiol. 64(22), 2330-2339 (2014).

11. Yoon SH, Bleiziffer S, De Backer O et al. Procedural and clinical outcomes in transcatheter aortic valve replacement for bicuspid versus tricuspid aortic valve stenosis. J. Am. Coll. Cardiol. 69(21), 2579-2589 (2017).

12. Perlman GY, Blanke P, Dvir D et al. Bicuspid aortic valve stenosis: favorable early outcomes with a next-generation transcatheter heart valve in a multicenter study. JACC Cardiovasc. Interv. 9(8), 817-824 (2016).

13. Latib A, Naganuma T, Abdel-Wahab M et al. Treatment and clinical outcomes of transcatheter heart valve thrombosis. Circ. Cardiovasc. Interv. 8(4), e001779 (2015).

14. Makkar RR, Fontana G, Jilaihawi $\mathrm{H}$ et al. Possible subclinical leaflet thrombosis in bioprosthetic aortic valves. N. Engl. J. Med. 373(21), 2015-2024 (2015).

15. Pache G, Schoechlin S, Blanke P et al. Early hypo-attenuated leaflet thickening in balloon-expandable transcatheter aortic heart valves. Eur. Heart J. 37(28), 2263-2271 (2016).

16. Leetmaa T, Hansson NC, Leipsic J et al. Early aortic transcatheter heart valve thrombosis: diagnostic value of contrast-enhanced multidetector computed tomography. Circ. Cardiovasc. Interv. 8(4), e001596 (2015). 
17. Chakravarty T, Abramowitz Y, Jilaihawi H, Makkar RR. Leaflet motion abnormality after TAVI: genuine threat or much ado about nothing? EuroIntervention 12(Y), Y28-Y32 (2016).

18. Barbanti M, Tamburino C. Optimisation of TAVI: is it mature enough to be defined as a PCI-like procedure? EuroIntervention 11(Suppl. W), W110-W113 (2015).

19. Barbanti M, Todaro D, Costa G et al. Optimized screening of coronary artery disease with invasive coronary angiography and ad hoc percutaneous coronary intervention during transcatheter aortic valve replacement. Circ. Cardiovasc. Interv. 10(8), e005234 (2017).

20. Barbanti M, Capranzano P, Ohno Y et al. Early discharge after transfemoral transcatheter aortic valve implantation. Heart 101(18), 1485-1490 (2015).

21. Durand E, Eltchaninoff $\mathrm{H}$, Canville A et al. Feasibility and safety of early discharge after transfemoral transcatheter aortic valve implantation with the Edwards SAPIEN-XT prosthesis. Am. J. Cardiol. 115(8), 1116-1122 (2015).

22. Jensen HA, Condado JF, Devireddy C et al. Minimalist transcatheter aortic valve replacement: the new standard for surgeons and cardiologists using transfemoral access? J. Thorac. Cardiovasc. Surg. 150(4), 833-839 (2015).

23. Abramowitz $\mathrm{Y}$, Jilaihawi $\mathrm{H}$, Chakravarty $\mathrm{T}$ et al. Feasibility and safety of balloon-expandable transcatheter aortic valve implantation with moderate or without predilatation. EuroIntervention 11(10), 1132-1139 (2016).

24. Arsalan M, Walther T. Durability of prostheses for transcatheter aortic valve implantation. Nat. Rev. Cardiol. 13(6), 360-367 (2016).

25. Barbanti M, Tamburino C. Late degeneration of transcatheter aortic valves: pathogenesis and management. EuroIntervention 12(Y), Y33-Y36 (2016).

26. Barbanti M, Webb JG, Tamburino C et al. Outcomes of Redo Transcatheter Aortic Valve Replacement for the Treatment of Postprocedural and Late Occurrence of Paravalvular Regurgitation and Transcatheter Valve Failure. Circ. Cardiovasc. Interv. 9(9), e003930 (2016).

27. Capodanno D, Petronio AS, Prendergast B et al. Standardized definitions of structural deterioration and valve failure in assessing long-term durability of transcatheter and surgical aortic bioprosthetic valves: a consensus statement from the European Association of Percutaneous Cardiovascular Interventions (EAPCI) endorsed by the European Society of Cardiology (ESC) and the European Association for Cardio-Thoracic Surgery (EACTS). Eur. J. Cardiothorac. Surg. 52(3), 408-417 (2017). 